\title{
The cone conjecture for some rational elliptic threefolds
}

\author{
Arthur Prendergast-Smith
}

A central problem of modern minimal model theory is to describe the various cones of divisors associated to a projective variety. For Fano varieties the nef cone and movable cone are rational polyhedral by the cone theorem [4, Theorem 3.7] and the theorem of BirkarCascini-Hacon-McKernan [1]. For more general varieties the picture is much less clear: these cones need not be rational polyhedral, and can even have uncountably many extremal rays.

The Morrison-Kawamata cone conjecture [8, 3, 13] describes the action of automorphisms on the cone of nef divisors and the action of pseudo-automorphisms on the cone of movable divisors, in the case of a Calabi-Yau variety, a Calabi-Yau fibre space, or a Calabi-Yau pair. Although these cones need not be rational polyhedral, the conjecture predicts that they should have a rational polyhedral fundamental domain for the action of the appropriate group. It is not clear where these automorphisms or pseudo-automorphisms should come from; nevertheless, the conjecture has been proved in various contexts by Sterk-LooijengaNamikawa [11, 9] Kawamata [3], and Totaro [14.

In this paper we give some new evidence for the conjecture, by verifying it for some threefolds which are blowups of $\mathbf{P}^{3}$ in the base locus of a net (that is, a 2-dimensional linear system) of quadrics. Our main result is the following:

Theorem 0.1 Let $X$ be the blowup of $\mathbf{P}^{3}$ in 8 distinct points which are the base locus of a net of quadrics.

(1) The nef cone $\overline{A(X)}$ is rational polyhedral and spanned by effective divisors.

(2) If the net has no reducible member, the effective movable cone $\overline{M(X)}^{e}$ has a rational polyhedral fundamental domain for the action of PsAut $(X)$.

In Section 2 we will see that for any net of quadrics in $\mathbf{P}^{3}$ with 8 distinct basepoints, the blowup of the base locus of the net has an elliptic fibration over $\mathbf{P}^{2}$. The condition that the net have no reducible member is equivalent to the generic fibre of the fibration (an elliptic curve over the function field of $\mathbf{P}^{2}$ ) having Mordell-Weil rank 7, the maximum possible. Although in statement (2) we restrict to this class of nets, much of the proof works for general nets, and it should be possible to fill in the remaining details.

The proof of Theorem 0.1 relies to a large extent on the explicit geometry of nets of quadrics in $\mathbf{P}^{3}$ and so seems difficult to generalise to other classes of varieties. Nevertheless, the result is significant inasmuch as it seems to be the first verification of the cone conjecture for a klt Calabi-Yau pair $(X, \Delta)$ of dimension 3 with $\Delta \neq 0$. (See the next section for definitions.) This lends further support to the point of view that klt Calabi-Yau pairs provide a natural setting for the conjecture.

Thanks to Klaus Hulek and Burt Totaro for their comments. 


\section{The cone conjecture}

In this section we give the precise statement of the cone conjecture for klt Calabi-Yau pairs, following [14]. (See also Section 1 of [14] for history and examples.) We work throughout over an algebraically closed field $k$ of characteristic 0 . A rational polyhedral cone in a real vector space $V$ with a $\mathbf{Q}$-structure is a closed convex cone with finitely many extremal rays, each spanned by a rational vector.

Suppose $f: X \rightarrow S$ is a projective surjective morphism of normal varieties with connected fibres. A Cartier divisor $D$ on $X$ is said to be $f$-nef (resp. $f$-movable, $f$-effective) if $D \cdot C \geq 0$ for all curves $C$ mapped to a point by $f$ (resp. if codim Supp $\operatorname{Coker}\left(f^{*} f_{*} \mathcal{O}_{X}(D) \rightarrow \mathcal{O}_{X}(D)\right) \geq$ 2 , if $\left.f_{*} \mathcal{O}_{X}(D) \neq 0\right)$.

We define the real vector space $N^{1}(X / S)$ to be $\operatorname{Div}(X) / \cong_{S} \otimes \mathbf{R}$ where $\operatorname{Div}(X)$ is the group of Cartier divisors on $X$ and $\cong_{S}$ denotes numerical equivalence over $S$. We denote by $N^{1}(X / S)_{\mathbf{Z}}$ the free abelian group in $N^{1}(X / S)$ consisting of numerical classes of Cartier divisors. The $f$-nef cone $\overline{A(X / S)}$ (resp. closed $f$-movable cone $M(X / S), f$-pseudoeffective cone $B(X / S)$ ) is the closed convex cone generated by classes of $f$-nef (resp. $f$-movable, $f$ effective) divisors. The $f$-effective cone $B^{e}(X / S)$ is the cone generated by $f$-effective Cartier divisors. We denote by $\overline{A(X / S)}^{e}$ and $\overline{M(X / S)}^{e}$ the intersections $\overline{A(X / S)} \cap B^{e}(X / S)$ and $M(X / S) \cap B^{e}(X / S)$, and call them the $f$-effective $f$-nef cone and $f$-effective $f$-movable cone respectively.

Define a pseudo-isomorphism from $X_{1}$ to $X_{2}$ over $S$ to be a birational map $X_{1} \rightarrow X_{2}$ over $S$ which is an isomorphism in codimension 1. A small $\mathbf{Q}$-factorial modification (SQM) of $X$ over $S$ means a pseudo-isomorphism over $S$ from $X$ to another $Q$-factorial variety with a projective morphism to $S$.

For an $\mathbf{R}$-divisor $\Delta$ on a normal $\mathbf{Q}$-factorial variety $X$, the pair $(X, \Delta)$ is $k l t$ if, for all resolutions $\pi: \tilde{X} \rightarrow X$ with a simple normal crossing R-divisor $\tilde{\Delta}$ such that $K_{\tilde{X}}+\tilde{\Delta}=$ $\pi^{*}\left(K_{X}+\Delta\right)$, the coefficients of $\tilde{\Delta}$ are less than 1. (In particular if $X$ is smooth and $D$ is a smooth divisor on $X$, then $(X, r D)$ is klt for any $r<1$.) We say that $(X / S, \Delta)$ is a $k l t$ Calabi-Yau pair if $(X, \Delta)$ is a $\mathbf{Q}$-factorial klt pair with $\Delta$ effective such that $K_{X}+\Delta$ is numerically trivial over $S$.

We denote the groups of automorphisms or pseudo-automorphisms of $X$ over $S$ which preserve a divisor $\Delta$ by $\operatorname{Aut}(X / S, \Delta)$ and $\operatorname{PsAut}(X / S, \Delta)$. Note that the action of $\operatorname{Aut}(X / S, \Delta)$ and $\operatorname{PsAut}(X / S, \Delta)$ on $N^{1}(X / S)$ is determined by the images of the representations $\operatorname{Aut}(X / S, \Delta) \rightarrow$ $G L\left(N^{1}(X / S)_{\mathbf{Z}}\right)$ and $\operatorname{PsAut}(X / S, \Delta) \rightarrow G L\left(N^{1}(X / S)_{\mathbf{Z}}\right)$. We denote the images of these representations by $\operatorname{Aut}^{*}(X / S, \Delta)$ and $\operatorname{PsAut}^{*}(X / S, \Delta)$.

Conjecture 1.1 Let $(X / S, \Delta)$ be a klt Calabi-Yau pair. Then:

(1) The number of Aut $(X / S, \Delta)$-equivalence classes of faces of the effective nef cone $\overline{A(X / S)}$ corresponding to birational contractions or fibre space structures is finite. Moreover, there exists a finite rational polyhedral cone $\Pi$ which is a fundamental domain for the action of $A u t^{*}(X / S, \Delta)$ on $\overline{A(X / S)}$ in the sense that

(a) $\overline{A(X / S)}^{e}=A u t^{*}(X / S, \Delta) \cdot \Pi$,

(b) Int $\Pi \cap g$ Int $\Pi=\emptyset$ for $g \neq 1$ in $A u t^{*}(X / S, \Delta)$.

(2) The number of PsAut $(X / S, \Delta)$-equivalence classes of chambers ${\overline{A\left(X^{\prime} / S, \alpha\right)}}^{e}$ in the cone $\overline{M(X / S)}{ }^{e}$ corresponding to marked SQMs $f^{\prime}: X^{\prime} \rightarrow S$ of $X \rightarrow S$ with marking $\alpha$ : $X^{\prime} \rightarrow X$ is finite. Moreover, there exists a finite rational polyhedral cone $\Pi^{\prime}$ which is a fundamental domain for the action of PsAut $(X / S, \Delta)$ on $\overline{M(X / S)}^{e}$. 
The conjecture has been proved for Calabi-Yau surfaces by Looijenga-Sterk and Namikawa [11, 9], for klt Calabi-Yau pairs of dimension 2 by Totaro [14, and for Calabi-Yau fibre spaces of dimension 3 over a positive-dimensional base by Kawamata [3]. For Calabi-Yau 3-folds there are significant results by Oguiso-Peternell [10, Szendröi [12], Uehara [15], and Wilson [16], but the conjecture remains open.

\section{Nets of quadrics in $\mathrm{P}^{3}$}

In this section we give some relevant facts about blowups of $\mathbf{P}^{3}$ in the base locus of a net of quadrics and fix some notation. We then explain what the cone conjecture predicts in this situation.

If $X$ is the blowup of $\mathbf{P}^{3}$ in any set of 8 points $p_{1}, \ldots, p_{8}$, then $N^{1}(X)$ is 9-dimensional with basis $\left\{H, E_{1}, \ldots, E_{8}\right\}$, where $H$ is the pullback to $X$ of the hyperplane class on $\mathbf{P}^{3}$ and $E_{i}$ is the class of the exceptional divisor of the blowup of $p_{i}$. The dual vector space $N_{1}(X)$ has basis $\left\{l, l_{1}, \ldots, l_{8}\right\}$, where $l$ is the pullback to $X$ of the class of a line in $\mathbf{P}^{3}$ and $l_{i}$ is the class of a line in $E_{i}$. The intersection pairing between these spaces is specified by the following intersection numbers: $H \cdot l=1, H \cdot l_{i}=0, E_{i} \cdot l=0, E_{i} \cdot E_{j}=-\delta_{i j}$, for all $i$ and $j$.

Now suppose the 8 points are distinct and are the base locus of a net of quadrics in $\mathbf{P}^{3}$. The proper transforms of quadrics in the net are (up to scalar) sections of the line bundle $2 H-E_{1}-\ldots-E_{8}=-\frac{1}{2} K_{X}$ : since we have blown up the base locus of the net, $-\frac{1}{2} K_{X}$ is basepoint-free on $X$ and so gives a surjective morphism $f: X \rightarrow \mathbf{P}^{2}$. Since $f$ is given by sections of $-\frac{1}{2} K_{X}$ we have $-\frac{1}{2} K_{X}=f^{*}(L)$ for $L$ the hyperplane class on $\mathbf{P}^{2}$. This implies that $-\frac{1}{2} K_{X} \cdot C=0$ for any curve $C$ on $X$ mapped to a point by $f$. Adjunction therefore tells us that the smooth fibres of $f$ are curves with trivial canonical bundle, hence elliptic curves. In other words, $f: X \rightarrow \mathbf{P}^{2}$ is an elliptic fibration. If $X_{\eta}$ denotes the generic fibre of $f$, we define the Mordell-Weil rank $\rho$ of $f$ ( or of $X$ ) to be the rank of the finitely-generated abelian group $\operatorname{Pic}^{0}\left(X_{\eta}\right)$ of degree-0 line bundles on $X_{\eta}$. One can show [13, Theorem 7.2] that $\rho(f)=7-d$ where $d$ is the number of reducible quadrics (unions of 2 distinct planes) in the net.

The elliptic fibration $f$ on $X$ is important because it gives us a supply of pseudo-automorphisms of $X$. Using the group law on an elliptic curve, $\mathrm{Pic}^{0}\left(X_{\eta}\right)$ acts on $X_{\eta}$ by automorphisms and by [13, Lemma 6.2] this extends to an action on $X$ by pseudo-automorphisms. That is, we can identify $\operatorname{Pic}^{0}\left(X_{\eta}\right)$ with a subgroup of $\operatorname{PsAut}(X)$. (We will see in the course of the proof that this subgroup gives enough pseudo-automorphisms to verify the conjecture.) More precisely, since $f$ is given by sections of the line bundle $-\frac{1}{2} K_{X}$, the action of elements of $\operatorname{Pic}^{0}\left(X_{\eta}\right)$ preserves divisors $\Delta=\frac{1}{2} D$ for $D$ a smooth divisor in the linear system $\left|-2 K_{X}\right|$, and commutes with the morphism $f: X \rightarrow \mathbf{P}^{2}$, so we can identify $\operatorname{Pic}^{0}\left(X_{\eta}\right)$ with a subgroup of $\operatorname{PsAut}\left(X / \mathbf{P}^{2}, \Delta\right)$ for any $\Delta$ of this form.

We must also say something about the reducible fibres of $f$. Note that by our description of $f$, all fibres are isomorphic to quartic curves in $\mathbf{P}^{3}$ which are the complete intersection of 2 quadrics in the net. First suppose a reducible fibre contains a line $L$. It is easy to see that $L$ must be the line joining 2 basepoints $p_{i}$ and $p_{j}$ of the net, so its proper transform on $X$ has class $l-l_{i}-l_{j}$ in $N_{1}(X)$. We denote this class by $C_{i j}$.

We will see in due course that the classes $C_{i j}$ play an important role in the proof of Theorem 0.1. Note that there are $\left(\begin{array}{l}8 \\ 2\end{array}\right)=28$ such lines, each contained in exactly 1 fibre of $f$, and hence at most 28 fibres containing a line. 
If a reducible fibre does not contain a line, it is the union of 2 irreducible conics in $\mathbf{P}^{3}$. Each conic is contained in a plane, and the union of the planes is a reducible quadric in the net. We will denote the classes in $N^{1}(X)$ of the 2 components of the proper transform of a reducible quadric $Q_{i}$ in the net by $D_{i}^{a}(a=1,2)$. For any $i$ we have $D_{i}^{1}+D_{i}^{2}=-\frac{1}{2} K_{X}=f^{*}(L)$, so both components must be mapped by $f$ to a line $L_{i}$ in $\mathbf{P}^{2}$. For any point $p \in L_{i}$ the fibre $f^{-1}(p)$ is then a reducible curve, the union of 2 conics in $\mathbf{P}^{3}$, one contained in each component $D_{i}^{a}$ of $Q_{i}$. We denote the class in $N_{1}(X)$ of the (possibly reducible) curve $f^{-1}(p) \cap D_{i}^{a}$ by $F_{i}^{a}$.

It is easy to see that any such plane and any such conic must both contain exactly 4 basepoints $p_{q}, p_{r}, p_{s}, p_{t}$ of the net, so in terms of our bases for $N^{1}(X)$ and $N_{1}(X)$ their proper transforms have classes $D_{i}^{a}=H-E_{q}-E_{r}-E_{s}-E_{t}$ and $F_{i}^{a}=2 l-l_{q}-l_{r}-l_{s}-l_{t}$. By the intersection numbers given above we get $D_{i}^{a} \cdot F_{i}^{a}=-2$. Also if $F$ is the class of any fibre of $f$ we have $D_{i}^{a} \cdot F=0$ because $D_{i}^{a}$ maps to a line in $\mathbf{P}^{2}$. Since $F=F_{i}^{1}+F_{i}^{2}$ for any $i$ we get $D_{i}^{a} \cdot F_{i}^{b}=2$ for $a \neq b$.

Define a prime divisor $D$ on $X$ to be vertical if $f(D) \neq \mathbf{P}^{2}$. Since any divisor pulled back from $\mathbf{P}^{2}$ is a multiple of $-\frac{1}{2} K_{X}$, the description of the reducible fibres of $f$ shows that the only vertical divisors on $X$ have divisor class either a multiple of $-\frac{1}{2} K_{X}$ or else $D_{i}^{a}$, where the latter are effective. We will see that vertical divisors play an important role in describing the movable cone of $X$ : namely, Lemma 4.6 shows that the $f$-movable cone is more or less defined by intersection numbers with fibral curves lying inside vertical divisors. Note however that for the final steps of the proof, we restrict to the case of Mordell-Weil rank 7, which by the discussion above is equivalent to the fact that $X$ has no vertical divisors other than multiples of $-\frac{1}{2} K_{X}$.

We mention some facts about the birational geometry of $X$. Suppose $\phi: X \rightarrow X^{\prime}$ is some other projective variety obtained by flopping some $f$-fibral curves on $X$ (that is, curves contained in fibres of $f$ ). The line bundle $-\frac{1}{2} K_{X^{\prime}}$ is basepoint-free on $X^{\prime}$ and gives another elliptic fibration $f^{\prime}: X^{\prime} \rightarrow \mathbf{P}^{2}$ such that $f=f^{\prime} \circ \phi$ as rational maps. Also, $\phi$ induces an identification $\phi_{*}$ of the spaces $N^{1}(X)$ and $N^{1}\left(X^{\prime}\right)$ and hence an identification of the dual spaces $N_{1}(X)$ and $N_{1}\left(X^{\prime}\right)$. Therefore for any such $X^{\prime}$ we can think of the nef cone $\overline{A\left(X^{\prime}\right)}$ as a cone in the vector space $N_{1}(X)$, and the closed cone of curves $\overline{\operatorname{Curv}\left(X^{\prime}\right)}$ (the dual of the nef cone) as a cone in $N_{1}(X)$. Also note that $\phi_{*}$ identifies $K_{X}$ and $K_{X^{\prime}}$ and so the subspaces $K_{X}^{\perp}=\left\{x \in N_{1}(X): K_{X} \cdot x=0\right\}$ and $K_{X^{\prime}}^{\perp}=\left\{x \in N_{1}\left(X^{\prime}\right): K_{X} \cdot x=0\right\}$ are identified. We can therefore speak of the subspace $K^{\perp} \subset N_{1}(X)$ without reference to a particular model of $X$.

Now we explain the predictions of the cone conjecture in this situation. If $X$ is the blowup of the base locus of a net of quadrics, we saw that the line bundle $-\frac{1}{2} K_{X}$ is basepoint-free. Therefore $-2 K_{X}$ is basepoint-free also, so by Bertini's theorem a general divisor $D \in\left|-2 K_{X}\right|$ is smooth. As mentioned in the previous section the pair $\left(X, \frac{1}{2} D\right)$ is then klt, and $K_{X}+\frac{1}{2} D$ is numerically trivial (over $S=\operatorname{Spec} k$ ). The cone conjecture therefore predicts that the groups $\operatorname{Aut}^{*}\left(X, \frac{1}{2} D\right)$ and $\operatorname{PsAut}^{*}\left(X, \frac{1}{2} D\right)$ act on the cones $\overline{A(X)}^{e}$ and $\overline{M(X)}^{e}$ respectively with rational polyhedral fundamental domain. The first statement of Theorem 0.1 says that the prediction about the nef cone is true for all such $X$, in a strong sense: the nef cone itself is rational polyhedral. (The existence of a rational polyhedral fundamental domain then follows, as we will see in the next section.) The second statement of the theorem says that the prediction about the movable cone is also true, although (as we shall see) that cone itself is 'almost never' rational polyhedral. 


\section{Nef cones}

In this section we will prove the first statement of Theorem 0.1 , namely that if $X$ is the blowup of $\mathbf{P}^{3}$ in the base locus of a net of quadrics with 8 distinct basepoints, then $\overline{A(X)}$ is a rational polyhedral cone. In the case where $X$ has Mordell-Weil rank $\rho=7$, we prove the same thing about the nef cones of flops $X^{\prime}$ of $X$ which we will use in the next section.

The cone theorem [4, Theorem 3.7] says (in any dimension) that if $(X, \Delta)$ is a klt pair with $\Delta$ effective, any $\left(K_{X}+\Delta\right)$-negative extremal ray of $\overline{\operatorname{Curv}(X)}$ can be contracted to give a projective variety $Z$. In the case that $X$ is s smooth threefold and $\Delta=0$, the following theorem of Mori [7, Theorem 3.3, Theorem 3.5] gives the possibilities for the exceptional locus of the contraction:

Theorem 3.1 (Mori) Suppose that $X$ is a smooth projective threefold, and $f: X \rightarrow Z$ is the contraction morphism associated to a $K_{X}$-negative extremal ray of $\overline{C u r v(X)}$. Then either $\operatorname{dim} Z \leq 2$ and the anticanonical bundle $-K_{X}$ is $f$-ample, or else $f$ is birational, the exceptional set $\operatorname{Exc}(f)$ is a prime divisor $D$ on $X$, and the possibilities for $D$ and $f$ are as follows:

1. $D$ is a $\mathbf{P}^{1}$-bundle over a smooth curve $C$, and $f_{\mid D}$ is the bundle map $D \rightarrow C$,

2. $D \cong \mathbf{P}^{2}$ with normal bundle $\mathcal{O}_{D}(D) \cong \mathcal{O}_{\mathbf{P}^{2}}(-1)$, and $f$ contracts $D$ to a smooth point,

3. $D \cong \mathbf{P}^{1} \times \mathbf{P}^{1}$ with $\mathcal{O}_{D}(D)$ of bidegree $(-1,-1)$, and $f$ contracts $D$ to a point,

4. $D$ is isomorphic to a singular quadric in $\mathbf{P}^{3}$ with $\mathcal{O}_{D}(D)=\mathcal{O}_{D} \otimes \mathcal{O}_{\mathbf{P}^{3}}(-1)$, and $f$ contracts $D$ to a point,

5. $D \cong \mathbf{P}^{2}$ with normal bundle $\mathcal{O}_{D}(D) \cong \mathcal{O}_{\mathbf{P}^{2}}(-2)$, and $f$ contracts $D$ to a point.

Only one of these possibilities is relevant to us:

Proposition 3.2 Suppose $X$ is a threefold obtained by blowing up the base locus of a net of quadrics in $\mathbf{P}^{3}$, and let $R$ be a $K_{X}$-negative extremal ray of the closed cone of curves $\overline{C u r v(X)}$. Then the contraction morphism $\operatorname{cont}_{R}: X \rightarrow Z$ is birational of type 2 on the above list that is, the exceptional divisor $D$ is isomorphic to $\mathbf{P}^{2}$, with normal bundle $\mathcal{O}_{\mathbf{P}^{2}}(-1)$, and $D$ blows down to a smooth point. Moreover, the exceptional divisor $D$ in this case must be the exceptional divisor $E_{i}$ of the blowup of one of the basepoints $p_{i}$ of the net.

Proof: First let us show that $\operatorname{cont}_{R}$ must be birational. The key point is that $\operatorname{since~} \operatorname{cont}_{R}$ is the contraction of an extremal ray, all the curves contracted must be numerical multiples of each other.

First suppose $\operatorname{dim} Z=0$. Then all curves on $X$ are numerical multiples of each other, which is clearly false.

Next suppose $\operatorname{dim} Z=1$. Choose one of the exceptional divisors $E_{i}$. I claim that the morphism $\operatorname{cont}_{R}$ cannot contract any curve in $E_{i}$. For any such curve is a numerical multiple of $l_{i}$, therefore all curves contracted are numerical multiples of $l_{i}$, implying that $\operatorname{cont}_{R}$ is the contraction of $E_{i}$, which is birational, contradicting our hypothesis. So the restriction of $\operatorname{cont}_{R}$ to $E_{i}$ contracts no curve, therefore is a surjection $E_{i} \rightarrow Z$. But there can be no 
surjection from $E_{i} \cong \mathbf{P}^{2}$ onto a curve, since the fibres over distinct points would be disjoint curves in $\mathbf{P}^{2}$.

Next suppose $\operatorname{dim} Z=2$. As above we get $\left(\operatorname{cont}_{R}\right)_{\mid E_{i}}: E_{i} \rightarrow Z$, a map from $\mathbf{P}^{2}$ to a smooth surface which contracts no curves. As before the image cannot be a curve so it must have dimension 2: therefore $\left(\operatorname{cont}_{R}\right)_{\mid E_{i}}$ is surjective. Since $\operatorname{cont}_{R}$ contracts no curves, Stein Factorization [2, III, Corollary 11.5] shows that it is a finite morphism. So the pushforward $\operatorname{map}\left(\operatorname{cont}_{R}\right)_{*}: \operatorname{Pic}\left(E_{i}\right)_{\mathbf{Q}} \rightarrow \operatorname{Pic}(Z)_{\mathbf{Q}}$ is surjective, implying that $\rho(Z) \leq 1$. On the other hand, [7, Theorem 3.2] says that the contraction of an extremal ray lowers the Picard number by 1 , so $\rho(Z)=\rho(X)-1=8$, which is a contradiction.

So we may assume that $\operatorname{cont}_{R}$ is birational, and therefore given by one of the 5 possibilities on Mori's list. My claim is that only the second of these 5 cases can occur for $X$ the blowup of $\mathbf{P}^{3}$ in the base locus of a net of quadrics. To see this, we will use adjunction for each of the divisors $D$ above. (This is valid, since each $D$ is a normal divisor in a smooth variety.) For any curve $C$ contained in $D$, we have $K_{X} \circ C=\left(K_{X} \otimes \mathcal{O}_{D}\right) \circ C$, where the first product is in $C H^{*}(X)$ and the second in $C H^{*}(D)$. Adjunction then lets us write the second expression as $\left(K_{D}-D\right) \circ C$. Let us see what this gives in each of the 5 cases above, for some choice of $C$.

1. Fix a section $S_{0}$ of the bundle $D$, and let $C$ denote any fibre. Then one can show 2 , Corollary V.2.11] that $K_{D} \equiv-2 S_{0}+k C$, for some integer $k$. In particular, since $C^{2}=0$ we have $K_{D} \circ C=-2$. That gives $K_{X} \circ C=\left(K_{D}-D\right) \circ C=-2-D \circ C$.

On the other hand, $X$ is the blowing up of a smooth curve in $Z$, so we have $K_{X}=$ $f^{*}\left(K_{Z}\right)+\mathcal{O}_{X}(D)$. Since $C$ is contracted by $f$, we get $K_{X} \circ C=D \circ C$. Equating these expressions gives $-2-D \circ C=D \circ C$, hence $D \circ C=-1$. Therefore $K_{X} \circ C=-2+1=-1$. This is impossible in our case, since all the coefficients of $K_{X}$ with respect to the usual basis of $\operatorname{Pic}(X)$ are even, hence we must have $K_{X} \circ C \in 2 \mathbf{Z}$ for any curve $C$.

2. This case does occur for our varieties $X$ : blowing down any exceptional divisor $E_{i}$ (where $p_{i}$ is a basepoint with no infinitely near basepoints) gives an example. I claim these are the only examples: any extremal contraction $f: X \rightarrow Z$ to a smooth $Z$ with exceptional divisor $D \cong \mathbf{P}^{2}$ and $\mathcal{O}_{D}(D) \cong \mathcal{O}(-1)$ must have $D=E_{i}$ for some basepoint $p_{i}$.

To see this, suppose that $D$ is a divisor satisfying the above conditions, distinct from each of the $E_{i}$. I claim that $D$ must be disjoint from each $E_{i}$. To prove this, suppose $D$ is not disjoint from $E_{i}$ : then the intersection $D \cap E_{i}$ is a curve $\Gamma$. Since $f$ contracts $D$, it must contract the curve $\Gamma$, hence must contract all of $E_{i}$, since all curves in $E_{i}$ are numerically equivalent up to constant. Since $D$ is irreducible, this gives $D=E_{i}$. This contradicts our assumption, so we must have $D$ disjoint from $E_{i}$. So we can contract all the exceptional divisors $E_{i}$ without changing the isomorphism class of $D$ or the normal bundle of $D$. This gives an effective divisor $D_{0} \subset \mathbf{P}^{3}$ isomorphic to $\mathbf{P}^{2}$ with normal bundle $\mathcal{O}_{\mathbf{P}^{2}}(-1)$, which is impossible. So we must have $D=E_{i}$ for some basepoint $p_{i}$.

3. In this case we have $K_{D}=\mathcal{O}(-2,-2)$, hence $K_{D}-\mathcal{O}_{D}(D)=\mathcal{O}(-2,-2)-\mathcal{O}(-1,-1)=$ $\mathcal{O}(-1,-1)$. Let $C$ be a ruling of $D$ : then $K_{X} \circ C=\mathcal{O}(-1,-1) \circ C=-1$. Again this is impossible, since $K_{X}$ has even coefficients.

4. In this case $\mathcal{O}_{D}(D) \cong \mathcal{O}_{D} \otimes \mathcal{O}_{\mathbf{P}^{3}}(-1)$. We can compute $K_{D}$ using adjunction: viewing $D$ as a divisor in $\mathbf{P}^{3}$, we have $K_{D}=\left(K_{\mathbf{P}^{3}}+\mathcal{O}_{\mathbf{P}^{3}}(D)\right)_{\mid D}=(\mathcal{O}(-4)+\mathcal{O}(2))_{\mid D}=\mathcal{O}(-2)_{\mid D}$. 
So $K_{X \mid D}=K_{D}-\mathcal{O}_{D}(D)=(\mathcal{O}(-2)-\mathcal{O}(-1))_{\mid D}=\mathcal{O}(-1)_{\mid D}$. But then if $C$ is a ruling of the cone, we have $K_{X} \circ C=\mathcal{O}(-1)_{\mid D} \circ C=-1$. Again this is impossible, since $K_{X}$ has even coefficients.

5. Here $K_{D}=\mathcal{O}_{\mathbf{P}^{2}}(-3)$, and $\mathcal{O}_{D}(D)=\mathcal{O}_{\mathbf{P}^{2}}(-2)$. Let $C$ be a line in $D \cong \mathbf{P}^{2}$ : then $K_{X} \circ C=\left(K_{D}-D\right) \circ C=\mathcal{O}_{\mathbf{P}^{2}}(-1) \circ C=-1$. Again this is impossible, since $K_{X}$ has even coefficients.

So as claimed, the only possibility for the contraction of a $K$-negative extremal ray of $\overline{\operatorname{Curv}(X)}$ is the contraction of one of the exceptional divisors $E_{i}$.

QED

We can stretch this argument further:

Corollary 3.3 Let $X$ be as above, and suppose $X^{\prime}$ is a smooth $S Q M$ of $X$. Then the same conclusion holds as for $X$ : for any extremal ray $R$ of $\overline{C u r v\left(X^{\prime}\right)}$, the contraction morphism $\operatorname{cont}_{R}$ is of type 2 on Mori's list.

Proof: Any SQM $\alpha: X^{\prime} \rightarrow X$ with $X^{\prime}$ smooth induces an isomorphism $N^{1}(X)_{\mathbf{Z}} \cong N^{1}\left(X^{\prime}\right)_{\mathbf{Z}}$ which identifies $K_{X}$ and $K_{X^{\prime}}$. In particular $K_{X^{\prime}}$ is 2-divisible in $N^{1}\left(X^{\prime}\right) \mathbf{z}$. The proof of the previous proposition then applies again. QED

So suppose we have a smooth SQM $X^{\prime}$ of $X$, and two $K_{X^{\prime}}$-negative extremal rays $R_{1}, R_{2}$ of $\overline{\operatorname{Curv}\left(X^{\prime}\right)}$. By the proposition, the corresponding contractions blow down divisors $D_{1}$ and $D_{2}$ in $X^{\prime}$, each one a copy of $\mathbf{P}^{2}$. If $D_{1} \cap D_{2}$ was nonempty, it would be some curve $C$ say. But then all curves in $D_{1}$ and all curves in $D_{2}$ would be numerical multiples of $C$, which by the cone theorem implies that the contraction morphism associated to $R_{1}$ say must also contract $D_{2}$. This contradicts the conclusion of Theorem 3.1 that the exceptional locus is irreducible. We conclude that any set $D_{1}, \ldots, D_{n}$ of such divisors must be pairwise disjoint. Since each one has normal bundle $\mathcal{O}(-1)$ in $X^{\prime}$, this implies further that their classes in $N^{1}\left(X^{\prime}\right)$ are linearly independent. So we can perform a sequence of blowdowns $X^{\prime}=X_{0} \rightarrow X_{1} \rightarrow \cdots \rightarrow X_{n}$, where $X_{i}$ is the variety obtained by contracting $D_{1}, \ldots, D_{i}$. The Picard number drops by 1 at each stage, and $X_{n}$ must have Picard number at least 1, so we conclude the following:

Corollary 3.4 Suppose $X^{\prime}, D_{1}, \ldots, D_{n}$ are as above. Then $n \leq \rho\left(X^{\prime}\right)-1$. In particular, $\overline{\operatorname{Curv}\left(X^{\prime}\right)}$ has at most $\rho\left(X^{\prime}\right)-1=\rho(X)-1=8 K$-negative extremal rays.

Now let us restrict to the case where $X^{\prime}$ is an SQM obtained from $X$ by a sequence of flops. (In fact we will see in the next section that these are all the SQMs of $X$.) Since $-K_{X^{\prime}}$ is nef for any $X^{\prime}$ obtained from $X$ by a sequence of flops, the cone of curves $\overline{\operatorname{Curv}\left(X^{\prime}\right)}$ is contained in the closed halfspace $\left\{C \in N_{1}\left(X^{\prime}\right) \mid K_{X^{\prime}} \cdot C \leq 0\right\}$. So the only other extremal rays of $\overline{\operatorname{Curv}\left(X^{\prime}\right)}$ are those in the hyperplane $K^{\perp}$. The class of a curve in $X^{\prime}$ lies in this hyperplane if and only if the curve is $f^{\prime}$-fibral, and since the fibres of $f^{\prime}$ are 1-dimensional, there are only finitely many classes of such curves. (Indeed if $g: Y \rightarrow Z$ is any morphism with 1-dimensional fibres, there are only finitely many classes of $g$-fibral curves, because the class of a fibre has only finitely many decompositions in the monoid of effective classes in $N_{1}(Y) \mathbf{z}$.) All this strongly suggests that each of the nef cones $\overline{A\left(X^{\prime}\right)}$ should be rational polyhedral. However, it is a priori possible that the cone behaves strangely in a neighbourhood of $K^{\perp}$ yielding extremal rays which are not spanned by the class of any curve.

We prove that this bad behaviour does not occur for $X$ : in other words, that $X$ has rational polyhedral cone of curves. Under the additional assumption that the Mordell-Weil rank $\rho$ is 
7 (or equivalently that the net has no reducible member) we prove the same conclusion for any $X^{\prime}$ obtained from $X$ by flopping a set of fibral curves.

Theorem 3.5 Suppose $X$ is the blowup of $\mathbf{P}^{3}$ in the base locus of a net of quadrics with 8 distinct basepoints. Then the closed cone of curves $\overline{C u r v(X)}$ is rational polyhedral, spanned by the classes $l_{i}$ of lines in the exceptional divisors $E_{i}$ together with the 28 classes $C_{i j}$. If in addition the net has Mordell-Weil rank $\rho=7$ then $\overline{C u r v\left(X^{\prime}\right)}$ is rational polyhedral for any $X^{\prime}$ obtained from $X$ by flopping a set of fibral curves.

Dually, the nef cones $\overline{A(X)}$ and $\overline{A\left(X^{\prime}\right)}$ are rational polyhedral in the situations described.

Proof: Corollary 3.4 showed that $\overline{\operatorname{Curv}(X)}$ has only finitely many $K$-negative extremal rays, so it suffices to show there are only finitely many extremal rays in $K^{\perp}$.

Consider a divisor class of the form $D_{i j}=H-E_{i}-E_{j}$ on $X$. This class is represented by the proper transform on $X$ of any plane in $\mathbf{P}^{3}$ passing through the points $p_{i}$ and $p_{j}$ so its base locus is the curve $C_{i j}$. Therefore if $C$ is any irreducible curve on $X$ which is not one of the curves $C_{i j}$, we must have $D_{i j} \cdot C \geq 0$. So all but finitely many irreducible curves $C$ on $X$ satisfy $D_{i j} \cdot C \geq 0$ for all $i, j$. In particular, any limit ray $R$ of a sequence of irreducible curves which is not contained in the cone spanned by the $C_{i j}$ must satisfy $D_{i j} \cdot R \geq 0$ for all $i, j$. We know all the extremal rays of $\overline{\operatorname{Curv}(X)}$ except those in $K^{\perp}$, so any other extremal ray $R$ must also satisfy $K_{X} \cdot R=0$. By computation the cone defined by the inequalities $D_{i j} \cdot C \geq 0$ and $K_{x} \cdot C=0$ is spanned by a finite set of vectors of the form $n l-(n-1) l_{i_{1}}-l_{i_{2}}-\cdots-l_{i_{n+2}}$, for $n=2, \ldots, 6$. Therefore this cone is contained in the cone $\mathbf{R}_{+}\left\{C_{i j}\right\}$ spanned by the classes $C_{i j}$. This proves that $\overline{\operatorname{Curv}(X)} \cap K^{\perp}=\mathbf{R}_{+}\left\{C_{i j}\right\}$, and therefore $\overline{\operatorname{Curv}(X)}$ is a rational polyhedral cone whose extremal rays are spanned by the classes $l_{i}$ and $C_{i j}$. This proves the first claim.

Now suppose $\rho=7$, and let us prove the claim about the cones $\overline{\operatorname{Curv}\left(X^{\prime}\right)}$. The idea is similar to the proof of the claim about $\overline{\operatorname{Curv}(X)}$, but the role of the divisors $D_{i j}$ is now played by an infinite set of movable divisors. Again Corollary 3.4 tells us that $\overline{\operatorname{Curv}\left(X^{\prime}\right)}$ has only finitely many $K$-negative extremal rays, so it suffices to prove that there are only finitely many extremal rays in $K^{\perp}$.

Recall that in general the action of $\operatorname{Pic}^{0}\left(X_{\eta}\right)$ on $N^{1}(X)$ is given by the formula $\psi_{y}(x)=$ $x+(x \cdot F) y+V(x, y)$, where $x \in N^{1}(X), y \in \operatorname{Pic}^{0}\left(X_{\eta}\right)$, and $V(x, y)$ is a vertical divisor. In the case $\rho=7$, the only vertical divisors are multiples of $-\frac{1}{2} K_{X}$, so we get $\psi_{y}(x)=$ $x+(x \cdot F) y+m\left(-\frac{1}{2} K_{X}\right)$. In particular if $x=D_{i j}$ and $y=n\left(E_{k}-E_{l}\right)$ we have $D_{i j} \cdot F=2$ and hence $\psi_{y}\left(D_{i j}\right)=H-E_{i}-E_{j}+2 n\left(E_{k}-E_{l}\right)+m\left(-\frac{1}{2} K_{X}\right)$. Also, since the base locus of $D_{i j}$ is the curve $C_{i j}$ and $\operatorname{Pic}^{0}\left(X_{\eta}\right)$ acts by pseudo-automorphisms over $\mathbf{P}^{2}$, the base locus of any such divisor $\psi_{y}\left(D_{i j}\right)$ is a finite union of fibral curves. If we then flop some fibral curves to obtain $X^{\prime}$, the base locus of the proper transform $\psi_{y}\left(D_{i j}\right)^{\prime}$ is again a finite union of fibral curves. The upshot is that for any irreducible curve $C$ on $X^{\prime}$, either $\psi_{y}\left(D_{i j}\right)^{\prime} \cdot C \geq 0$ for all $i, j$ and all $y$ in $\operatorname{Pic}^{0}\left(X_{\eta}\right)$, or else $C$ is one of the finitely many classes of fibral curves on $X^{\prime}$.

Now suppose $R$ is an extremal ray of $\overline{\operatorname{Curv}\left(X^{\prime}\right)}$ which lies in the subspace $K^{\perp}$. As before, any limit ray $R$ of a sequence of irreducible curves which is not in the cone spanned by the classes of fibral curves must satisfy $\psi_{y}\left(D_{i j}\right)^{\prime} \cdot R \geq 0$ for all $i, j$ and all $y$ in $\operatorname{Pic}^{0}\left(X_{\eta}\right)$. Suppose that $R$ is such a ray. Since $R \subset K^{\perp}$, any class $C$ which spans $R$ has $K \cdot C=0$, implying $\left(\psi_{y}\left(D_{i j}\right)\right)^{\prime} \cdot C=\left(D_{i j}+2 y\right)^{\prime} \cdot C \geq 0$ for any $x \in N^{1}(X)$ and $y \in \operatorname{Pic}^{0}\left(X_{\eta}\right)$. In particular if we put $x=D_{i j}$ and $y=n\left(E_{k}-E_{l}\right)$ we get $\left(\left(H-E_{i}-E_{j}\right)+2 n\left(E_{k}-E_{l}\right)\right)^{\prime} \cdot C \geq 0$ for all indices $i, j, k, l$ and all integers $n$. Now if $C=a l+\sum_{i} b_{i} l_{i}$ with coefficients $b_{i}$ not all equal, then $\left(E_{k}-E_{l}\right)^{\prime} \cdot C<0$ for some $k$ and $l$, implying $\left(\left(H-E_{i}-E_{j}\right)+2 n\left(E_{k}-E_{l}\right)\right)^{\prime} \cdot C<0$ 
for some indices $i, j, k, l$ and $n$ sufficiently large. This contradicts our choice of $R$. So the only possibility is that all coefficients $b_{i}$ are equal, which implies that $R$ is the ray spanned by $4 l-\sum_{i} l_{i}$, the class of a fibre. We conclude that $\overline{\operatorname{Curv}\left(X^{\prime}\right)} \cap K^{\perp}$ is spanned by the classes of fibral curves, which are finite in number, and therefore that $\overline{\operatorname{Curv}\left(X^{\prime}\right)}$ is a rational polyhedral cone, as claimed. QED

The cone conjecture concerns the nef effective cone $\overline{A(X)}^{e}$ rather than the whole nef cone. However, in our situation these cones coincide:

Proposition 3.6 Suppose $X$ is the blowup of $\mathbf{P}^{3}$ in the base locus of a net of quadrics with 8 distinct basepoints, and $X^{\prime}$ is obtained from $X$ by a sequence of flops of fibral curves. Then any nef divisor on any of the varieties $X^{\prime}$ is semi-ample, hence effective. In other words, $\overline{A\left(X^{\prime}\right)}{ }^{e}=\overline{A\left(X^{\prime}\right)}$ for all such $X^{\prime}$.

Proof: Since $\overline{A\left(X^{\prime}\right)}$ is rational polyhedral it suffices to prove that any integral divisor in $\overline{A\left(X^{\prime}\right)}$ is effective. The proposition is immediate for multiples of $-\frac{1}{2} K_{X^{\prime}}$ so assume $D$ is a nef integral divisor which is not such a multiple. By the Basepoint-Free theorem [4, Theorem 3.3] it suffices to show that the divisor $D-\frac{1}{2} K_{X^{\prime}}$ is big, which by the numerical criterion for bigness of nef divisors [5, Theorem 2.2.16] is equivalent to $\left(D-\frac{1}{2} K_{X^{\prime}}\right)^{3}>0$. Now $\left(-\frac{1}{2} K_{X^{\prime}}\right)^{3}=0$ and $D^{3} \geq 0$ since $D$ is nef; also $D^{2} \cdot\left(-\frac{1}{2} K_{X^{\prime}}\right) \geq 0$ since $D^{2} \in \overline{\operatorname{Curv}\left(X^{\prime}\right)}$. So it suffices to prove that $D \cdot\left(-\frac{1}{2} K_{X^{\prime}}\right)^{2}>0$. But $\left(-\frac{1}{2} K_{X^{\prime}}\right)^{2}$ is the class $F$ in $N^{1}\left(X^{\prime}\right)$ of any fibre of $f^{\prime}$. If $D \cdot F=0$ then since $D$ is nef we must have $D \cdot C=0$ for $C$ the class of any $f^{\prime}$-fibral curve. The classes of such curves span the codimension-1 subspace $K^{\perp}$ of $N_{1}\left(X^{\prime}\right)$ so $D$ is a multiple of $-\frac{1}{2} K_{X^{\prime}}$, contradicting our initial assumption. Therefore $D \cdot\left(-\frac{1}{2} K_{X^{\prime}}\right)^{2}>0$ as required. QED

We have proved the first statement of Theorem 0.1 , namely that $\overline{A(X)}$ is a rational polyhedral cone spanned by effective divisors. However, the first prediction of Conjecture 1.1 does not seem to follow immediately. The conjecture predicted there should be a rational polyhedral fundamental domain for the action of $\operatorname{PsAut}^{*}(X, \Delta)$ on $\overline{A(X)}$. (Recall that $\Delta$ is a Q-divisor $\frac{1}{2} D$ for some smooth member $D$ of the linear system $\left|-2 K_{X}\right|$.) To verify that statement for $X$, we use the following theorem of Looijenga [6, Proposition 4.1, Application 4.15]. (We state a stronger form than we need at present, for use in the next section.)

Theorem 3.7 (Looijenga) Let $V$ be a real vector space with $\mathbf{Z}$-structure and $C$ a strictly convex open cone in $V$ with nonempty interior. Let $G$ be a subgroup of $G L\left(V_{\mathbf{Z}}\right)$ which preserves $C$. Suppose there is a rational polyhedral cone $U$ in $\bar{C}$ such that $G \cdot U$ contains $C$. Then $G \cdot U$ is equal to the convex hull $C_{+}$of the rational points in $\bar{C}$, and there exists a rational polyhedral fundamental domain for the action of $G$ on $C_{+}$.

Corollary 3.8 The first statement of Conjecture 1.1 holds for $X$ : there is a rational polyhedral fundamental domain for the action of $A u t^{*}(X, \Delta)$ on $\overline{A(X)}^{e}$.

Proof: We have just seen that $\overline{A(X)}^{e}=\overline{A(X)}$, a rational polyhedral cone. Applying Theorem 3.7 with $\bar{C}=U=\overline{A(X)}$ and $G=\operatorname{Aut}^{*}(X, \Delta)$ we get the result. QED

Corollary 3.9 For $X$ as above, the group Aut $(X)$ is finite.

Proof: The cone $\overline{A(X)}$ is a rational polyhedral cone preserved by $\operatorname{Aut}^{*}(X)$. I claim that an infinite subgroup $G$ of $G L\left(N^{1}(X)_{\mathbf{Z}}\right)$ cannot preserve a strictly convex rational polyhedral 
cone with nonempty interior. For the action of any element of $g \in G$ must permute the primitive integral vectors in the extremal rays of the cone, and this permutation determines the action of $g$. So $G$ is realised as a subgroup of a finite permutation group. QED

\section{Movable cone}

The aim of this section is to prove the second part of Theorem 0.1; if $X$ is the blowup of $\mathbf{P}^{3}$ in the base locus of a net of quadrics with 8 distinct basepoints, and the Mordell-Weil rank $\rho(X)$ equals 7 , then there is a rational polyhedral fundamental domain for the action of PsAut* $(X)$ on $\overline{M(X)}^{e}$.

We remark that the second part of Theorem 0.1 is more difficult than the first: in particular, the effective movable cone $\overline{M(X)}^{e}$ is in general not rational polyhedral in the context we are considering. To see this, recall from the introduction that the Mordell-Weil group $\operatorname{Pic}^{0}\left(X_{\eta}\right)$ of the generic fibre of $f$ acts on $X$ by pseudo-automorphisms. I claim that the representation $\operatorname{Pic}^{0}\left(X_{\eta}\right) \rightarrow G L\left(N^{1}(X)_{\mathbf{Z}}\right)$ is faithful. To see this, note that any rational section $D$ of $f$ has an open subset covered by rational curves $C$ with $D \cdot C=-1$ (images under the section of lines in $\mathbf{P}^{2}$ ). If $D_{0}$ and $D_{1}$ are different rational sections, then the element $D_{1}-D_{0} \in \operatorname{Pic}^{0}\left(X_{\eta}\right)$ maps $D_{0}$ to $D_{1}+V$ where $V$ is an effective divisor pulled back from $\mathbf{P}^{2}$. Then for all but finitely many curves $C \subset D_{0}$ we have $\left(D_{1}+V\right) \cdot C \geq 0$, so $D_{1}+V$ and $D_{0}$ are numerically distinct, and therefore $D_{1}-D_{0}$ is not in the kernel of the representation. This proves the claim, and we conclude that if the Mordell-Weil group is infinite, then so too is its image in $G L\left(N^{1}(X)_{\mathbf{z}}\right)$. Pseudo-automorphisms of $X$ preserve the effective movable cone $\overline{M(X)}^{e}$, and (as mentioned before) an infinite subgroup of $G L\left(N^{1}(X) \mathbf{z}\right.$ ) cannot act on a strictly convex rational polyhedral cone with nonempty interior. Therefore the effective movable cone cannot be rational polyhedral unless the Mordell-Weil group is finite (or equivalently, as explained in Section 2, the net contains the maximum number 7 of reducible members).

The structure of the proof is as follows. First we show in Proposition 4.2 that the cone $\overline{M(X)}^{e}$ decomposes as the union of nef effective cones of SQMs of $X$ which are obtained by flopping curves in the fibres of $f: X \rightarrow \mathbf{P}^{2}$, and the interiors of these nef cones are disjoint. Moreover (Lemma 4.3), pseudo-automorphisms of $X$ act by permuting the cones. We saw in the previous section (under the assumption of maximum Mordell-Weil rank) that each of the nef cones is rational polyhedral, so it seems reasonable that some finite union of these cones might provide the fundamental domain we seek. Precisely, by Theorem 3.7, it is enough to show that the translates by pseudo-automorphisms of a finite union of these cones covers the effective movable cone.

To prove this, we again use the elliptic fibration structure on $X$. As previously mentioned, the Mordell-Weil group $\operatorname{Pic}^{0}\left(X_{\eta}\right)$ of the generic fibre of the fibration is a subgroup of the pseudo-automorphism group of $X$. We study the action of this subgroup on the quotient space $N^{1}\left(X / \mathbf{P}^{2}\right)$. By general results of Kawamata on 3-dimensional elliptic fibrations (Lemma 4.5), this action is easy to understand explicitly. Also, in Lemma 4.6 we are able to compute 'by hand' the relative movable cone, using the explicit geometry of the fibration.

Putting these facts together we find in Lemma 4.7 a rational polyhedral cone in the relative movable cone whose $\operatorname{Pic}^{0}\left(X_{\eta}\right)$-translates cover the whole cone. The key point of our method is to lift this action to the absolute movable cone. In Theorem 4.9 to find a rational polyhedral cone in $N^{1}(X)$ whose $\operatorname{Pic}^{0}\left(X_{\eta}\right)$-translates cover the whole effective movable cone. Since 
the Mordell-Weil group is a subgroup of the pseudo-automorphism group, the $\operatorname{PsAut}(X)$ translates of that rational polyhedral cone also cover the whole effective cone, as required.

As a final remark before starting the proof, we re-emphasise that most of our proof is valid for nets of arbitrary Mordell-Weil rank. We only impose the restriction to nets of maximum rank starting from Lemma 4.8. It seems likely that the remaining details can be filled in to give a complete proof for nets of arbitrary rank. The proof below would be simplified if we restricted to nets of maximum rank from the start; however, we retain the general argument as far as possible, to illustrate the fact that our methods still give a good deal of information in the general case.

Now let us begin the proof. The first step is to show that any movable divisor on $X$ can be made nef by a sequence of flops. For $D$ a $\mathbf{Q}$-Cartier $\mathbf{Q}$-divisor on a normal projective variety $Y$, define a $D$-flopping contraction of $Y$ to be a proper birational morphism $f: Y \rightarrow Z$ to a normal variety $Z$ such that the exceptional set of $f$ has codimension at least 2 in $Y$, the canonical class $K_{Y}$ is numerically $f$-trivial, and $-D$ is $f$-ample. The $D$-flop of $f$ is then defined to be the $\left(K_{Y}+D\right)$-flip of $f$. We need the following result [4, Theorem 6.14, Corollary 6.19]:

Lemma 4.1 Suppose $Y$ is a threefold with terminal singularities and $f: Y \rightarrow Z$ a $D$-flopping contraction (for some $\mathbf{Q}$-divisor D). Then the D-flop of $f$ exists. Moreover, any sequence of extremal D-flops on a terminal threefold is finite.

Here an extremal flop is one for which the flopping contraction has relative Picard number 1. In particular if $(X, \Delta)$ is a $\mathbf{Q}$-factorial klt pair with $\Delta$ effective and $f$ is the contraction of a $\left(K_{X}+\Delta\right)$-negative extremal ray, it is extremal, because all the curves contracted are numerical multiples of each other, by the cone theorem.

This lemma enables us to find the desired decomposition of the movable cone of $X$ into nef cones:

Proposition 4.2 The effective movable cone $\overline{M(X)}^{e}$ decomposes as a union of nef cones of SQMs of $X$ :

$$
\overline{M(X)}^{e}=\bigcup \overline{A\left(X^{\prime}, \alpha\right)}
$$

where the union on the right hand side is over all SQMs $\alpha: \underline{X^{\prime} \rightarrow-X}$. All these SQMs are obtained by flopping fibral curves. The interiors of the cones $\overline{A\left(X^{\prime}, \alpha\right)}$ are disjoint.

Proof: Suppose $D \in \overline{M(X)}^{e}$ is an effective Q-divisor on $X$ which is not nef. We know a movable divisor cannot be negative on a $K$-negative extremal ray, so the same is true of a divisor in $\overline{M(X)}$. So the description of $\overline{\operatorname{Curv}(X)}$ tells us that $D \cdot C_{i j}<0$ for some $i, j$. Choosing some $\epsilon>0$ sufficiently small, the cone theorem for the klt pair $(X, \epsilon D)$ tells us that the contraction of $C_{i j}$ exists. By Lemma 4.1 we can perform a flop to obtain a variety $X^{\prime}$ on which $D^{\prime} \cdot C^{\prime}>0$, where $D^{\prime}$ is the proper transform of $D$, and $C^{\prime}$ the cocentre of the flop. If $D^{\prime}$ is now nef we can stop. If not then $D^{\prime}$ is negative on some extremal ray of $\overline{\operatorname{Curv}\left(X^{\prime}\right)}$. Again $D^{\prime}$ cannot be negative on a $K$-negative extremal ray so $D^{\prime} \cdot R<0$ for some extremal ray $R \in K^{\perp}$. Choosing $\epsilon^{\prime}>0$ sufficiently small again the cone theorem for $\left(X^{\prime}, \epsilon^{\prime} D^{\prime}\right)$ tells us that $R$ is spanned by the class of a curve $C^{\prime}$ (necessarily a component of a fibre) and the $D^{\prime}$-flop exists. Continuing in this way, the lemma above guarantees that this process terminates at some finite stage: that is, the proper transform of $D$ becomes nef after some finite sequences of flops of fibral curves. 
If $D$ is not a $\mathbf{Q}$-divisor, the same argument works by adding at each stage a sufficiently small ample $\mathbf{R}$-divisor $D_{1}$ so that $D^{\prime}+D_{1}$ is a $\mathbf{Q}$-divisor which has negative degree on the same fibral curves as $D^{\prime}$.

We have shown that any effective movable divisor belongs to one of the effective nef cones ${\overline{A\left(X^{\prime}\right)}}^{e}$ where $X^{\prime}$ is obtained from $X$ by flopping fibral curves. So we have the inclusion $\overline{M(X)}^{e} \subset \cup{\overline{A\left(X^{\prime}, \alpha\right)}}^{e}$. The reverse inclusion is clear, since an ample divisor on $X^{\prime}$ is movable on $X$, so taking closures and intersecting with the effective cone we get $\cup{\overline{A\left(X^{\prime}, \alpha\right)}}^{e} \subset \overline{M(X)}^{e}$. Finally by Proposition 3.6 we get the statement above.

To see that these flops give all the SQMs of $X$ up to isomorphism, suppose that $\beta: Y \rightarrow X$ is any SQM. By the argument above we have $\overline{A(Y, \beta)} \subset \cup \overline{A\left(X^{\prime}, \alpha\right)}$, therefore the ample cone of $Y$ must intersect the ample cone of one of the flops, say $\alpha_{i}: X_{i} \rightarrow X$. So there exists a divisor $D$ on $X$ such that $\alpha_{i *}^{-1} D$ and $\beta_{*}^{-1} D$ are ample on $X_{i}$ and $Y$ respectively. Therefore

$$
X_{i}=\operatorname{Proj} R\left(X_{i}, \alpha_{i *}^{-1} D\right) \cong \operatorname{Proj} R\left(Y, \beta_{*}^{-1} D\right)=Y
$$

and the isomorphism is compatible with $\alpha_{i}$ and $\beta$.

Finally, the same argument applied to 2 SQMs $\alpha_{1}: X_{1} \rightarrow X$ and $\alpha_{2}: X_{2} \rightarrow X$ shows that the interiors of the cones $\overline{A\left(X_{1}, \alpha_{1}\right)}$ and $\overline{A\left(X_{2}, \alpha_{2}\right)}$ are disjoint in $\overline{M(X)}{ }^{2}$. QED

This decomposition of the movable cone is compatible with the action of pseudo-automorphisms:

Lemma 4.3 The group PsAut $(X)$ acts on $\overline{M(X)}^{e}$ by permuting the nef cones of the small modifications of $X$. More precisely suppose $\phi \in P s A u t(X)$ and $\alpha: Y \rightarrow X$ is an SQM of $X$. Then $\phi_{*}(\overline{A(Y)})=\overline{A\left(Y^{\prime}\right)}$ for $\alpha^{\prime}: Y^{\prime} \rightarrow X$ some other $S Q M$ of $X$.

Proof: Suppose $\alpha: Y \rightarrow X$ is the marking of $Y$. Then $\alpha^{\prime}$ is the SQM given by $\phi \circ \alpha$ : $Y \rightarrow X$. To see this works take any divisor $D$ on $X$ such that $\alpha_{*}^{-1}(D)$ is nef on $Y$ : in other words $D$ belongs to $\overline{A(Y, \alpha)}$. Then putting $\Delta=\phi_{*}(D)$ we have $(\phi \circ \alpha)_{*}^{-1}(\Delta)=\alpha_{*}^{-1}(D)$ nef on $Y$. So $\Delta$ belongs to $\overline{A\left(Y, \alpha^{\prime}\right)}$ and $\phi_{*}$ maps $D$ to $\Delta$. This holds for any $D \in \overline{A(Y)}$ and so $\phi_{*}(\overline{A(Y, \alpha)}) \subset \overline{A\left(Y, \alpha^{\prime}\right)}$. Exchanging $\phi$ and $\phi^{-1}$ we get the result. QED

The next step in the proof is to study the action of pseudo-automorphisms (more precisely, the Mordell-Weil group) on the relative movable cone $\overline{M\left(X / \mathbf{P}^{2}\right)}{ }^{e}$. First we must understand the relationship between the vector spaces $N^{1}(X)$ and $N^{1}\left(X / \mathbf{P}^{2}\right)$. Recall that $N^{1}\left(X / \mathbf{P}^{2}\right)$ was defined as the space of Cartier divisors on $X$ with real coefficients modulo numerical equivalence over $\mathbf{P}^{2}$, where a divisor $D$ is numerically trivial over $\mathbf{P}^{2}$ if $D \cdot C=0$ for every curve $C$ which maps to a point on $\mathbf{P}^{2}$. Those curves span a subspace of $N_{1}(X)$, so dually there is a projection map $p$ from $N^{1}(X)$ to $N^{1}\left(X / \mathbf{P}^{2}\right)$. As a piece of notation, from now on we will write $[D]$ to denote the image $p(D) \in N^{1}\left(X / \mathbf{P}^{2}\right)$ of an element $D \in N^{1}(X)$.

The first important question is to characterise this projection map:

Lemma 4.4 The projection $p: N^{1}(X) \rightarrow N^{1}\left(X / \mathbf{P}^{2}\right)$ has 1-dimensional kernel spanned by the class of $-\frac{1}{2} K_{X}$.

Proof: A class $D \in N^{1}(X)$ maps to 0 in $N^{1}\left(X / \mathbf{P}^{2}\right)$ if and only if $D \cdot C=0$ for every fibral curve $C$ on $X$. The description of $\overline{\operatorname{Curv}(X)}$ in the previous section shows that the classes of fibral curves span the hyperplane $K^{\perp}$, so $D$ must be a multiple of $-\frac{1}{2} K_{X}$. QED

For later use we introduce some notation related to this projection: for a class $D \in N^{1}(X)$, we denote its image $p(D) \in N^{1}\left(X / \mathbf{P}^{2}\right)$ by $[D]$. 
The following lemma of Kawamata [3, Lemma 3.5] shows that the action of $\operatorname{Pic}^{0}\left(X_{\eta}\right)$ is easy to understand if we pass to a suitable quotient space of $N^{1}\left(X / \mathbf{P}^{2}\right)$.

Lemma 4.5 Let $V\left(X / \mathbf{P}^{2}\right)$ denote the subspace of vertical divisors of $f: X \rightarrow \mathbf{P}^{2}$ and $W\left(X / \mathbf{P}^{2}\right)$ the affine subquotient space $\left\{x \in N^{1}\left(X / \mathbf{P}^{2}\right) / V\left(X / \mathbf{P}^{2}\right): x \cdot F=1\right\}$. Then $\operatorname{Pic}^{0}\left(X_{\eta}\right)$ acts properly discontinuously on $W(X / S)$ as a group of translations, and has fundamental domain a rational polyhedron $\Pi$.

Next we compute the relative effective and movable cones. It turns out that the fibration $f$ determines these cones in the simplest way one could hope for. That is, certain curves in the fibres of $f$ give obvious classes in $N_{1}\left(X / \mathbf{P}^{2}\right)$ on which any $f$-effective or $f$-movable divisor must have nonnegative degree, and it turns out that these obvious classes actually suffice to determine the cones completely. The precise result is the following:

Lemma 4.6 Suppose $f: X \rightarrow \mathbf{P}^{2}$ is as before. For $i=1,2$ let $D_{i}^{a}$ (resp. $F_{i}^{a}$ ) denote the components of the reducible quadric $Q_{i}$ in the net (resp. components of the fibre $f^{-1}\left(\eta_{i}\right)$ where $\eta_{i}$ is the generic point of $f\left(Q_{i}\right)$ ) and let $F$ denote the class of a fibre of $f$. Then

$$
\begin{aligned}
B^{e}\left(X / \mathbf{P}^{2}\right) & =\left\{x \in N^{1}\left(X / \mathbf{P}^{2}\right): x \cdot F>0\right\} \cup \mathbf{R}_{+}\left\{\left[D_{i}^{a}\right]\right\} \cup\{0\} \\
M\left(X / \mathbf{P}^{2}\right)^{e} & =\left\{x \in N^{1}\left(X / \mathbf{P}^{2}\right): x \cdot F>0, x \cdot F_{i}^{a} \geq 0 \text { for all } a, i\right\} \cup\{0\} .
\end{aligned}
$$

Note that two divisors whose classes are equal in $N^{1}\left(X / \mathbf{P}^{2}\right)$ differ by a multiple of $-\frac{1}{2} K_{X}$, so intersection numbers with all $f$-fibral curves are well-defined.

Proof: First suppose that $D$ is an $f$-effective Cartier divisor with degree $k \leq 0$ on the generic fibre. There exists a nonempty open set $U \subset \mathbf{P}^{2}$ such that $D\left(f^{-1}(U)\right) \neq 0$. Choose a nonzero section $s \in D\left(f^{-1}(U)\right)$. Then the class of the divisor $\Delta=\overline{\{s=0\}}$ differs from $D$ only on the codimension-1 subset $X \backslash f^{-1}(U)$. In particular these classes have the same degree $k$ on the generic fibre. Since $\Delta$ is effective this implies $k=0$. Moreover $k=0$ implies that $\Delta$ is a sum of vertical divisors so its class in $N^{1}(X)$ belongs to the cone $V$ spanned by $-\frac{1}{2} K_{X}$ and the $D_{i}^{a}$. Finally the divisor $\Delta-D$ is supported in $X \backslash f^{-1}(U)$ therefore its support maps onto a curve in $\mathbf{P}^{2}$. So any divisor in the support of $\Delta-D$ must also have class in the cone $V$. So for any $f$-effective class $D$ with degree $\leq 0$ on the generic fibre we can write $D=V_{1}-V_{2}$ where $V_{i}$ are classes in $V$. The image of $V$ in $N^{1}\left(X / \mathbf{P}^{2}\right)$ is the cone $\mathbf{R}_{+}\left\{\left[D_{i}^{a}\right]\right\}$, which is closed under negation since for any $i$ we have $\left[D_{i}^{1}\right]+\left[D_{i}^{2}\right]=0$ in $N^{1}\left(X / \mathbf{P}^{2}\right)$. Therefore $[D]=\left[V_{1}\right]-\left[V_{2}\right]$ belongs to this cone as claimed. This proves that the left-hand side of the first equation is contained in the right-hand side. To prove the reverse inclusion, first note that if a divisor $D$ has positive degree on an irreducible fibre $F$ then the restriction $D_{\mid F}$ is ample hence effective. But standard results on semicontinuity of cohomology [2, Corollary III.12.9] show that any section of $D_{\mid F}$ is the restriction of a section in $D\left(f^{-1}(U)\right)$ for $U \in \mathbf{P}^{2}$ some open subset. By definition that means $D$ is $f$-effective. Finally, all divisors in the cone $V$ are effective by definition hence $f$-effective, so all elements of $\mathbf{R}_{+}\left\{\left[D_{i}^{a}\right]\right\}$ lie in the $f$-effective cone. This completes the proof of the claim about the $f$-effective cone.

Now we must prove the claim about the $f$-movable cone. First note that if $D$ is a Cartier divisor in $N^{1}(X)$ with $D \cdot F_{i}^{a}<0$ for some $a$ and $i$, then $D$ cannot be $f$-movable. For suppose $C$ is a curve in $X$ with class $F_{i}^{a}$. If there was an open set $U \subset \mathbf{P}^{2}$ containing the point $f(C)$ and a section of $D\left(f^{-1}(U)\right)$ not vanishing identically along $C$ we would have $D \cdot C \geq 0$ contradicting our assumption: therefore every such curve $C$ is contained in 
Supp Coker $\left(f^{*} f_{*} \mathcal{O}_{X}(D) \rightarrow \mathcal{O}_{X}(D)\right)$. Since these curves $C$ fill up the divisor $D_{i}^{a}$ we conclude that $D$ cannot be $f$-movable. So the $f$-movable cone is contained in the cone $\left\{x \cdot F_{i}^{a} \geq\right.$ 0 for all $a, i\}$. If moreover $D$ is a nonzero $f$-effective $f$-movable class, it must have $D \cdot F>0$. For otherwise by the description of $B^{e}\left(X / \mathbf{P}^{2}\right)$ we would have $[D] \in \mathbf{R}_{+}\left\{\left[D_{i}^{a}\right]\right\}$. Any nonzero point in this cone has the form $\sum r_{a i}\left[D_{i}^{a}\right]$ with $r_{1 i}$ and $r_{2 i}$ not equal for all $i$. Say $r_{1 i}>r_{2 i}$ : then $[D] \cdot F_{i}^{1}<0$, contradicting our previous conclusion. So we have shown that left-hand side is contained in the right-hand side in the second equality above.

Conversely suppose that $x \in N^{1}\left(X / \mathbf{P}^{2}\right)$ satisfies $x \cdot F_{i}^{a} \geq 0$ for all $a, i$ and $x \cdot F>0$ : we want to show that $x$ belongs to the $f$-effective $f$-movable cone. First note that any such $x$ is $f$-effective by our description of the $f$-effective cone. Next suppose that $D$ is a divisor class with $D \cdot F_{i}^{a}>0$ for each $i$. Since $F=F_{i}^{1}+F_{i}^{2}$ for any $i$, the restriction of such a $D$ to any irreducible fibre is ample. Also since $D \cdot F_{i}^{a}>0$ for each $a$ and $i$ the restriction of $D$ to any component of a fibre not containing a line $C_{i j}$ is ample. So by taking a sufficiently large multiple $m D$ we get a line bundle whose restriction to all but finitely many fibres is very ample hence basepoint-free. Again by the semicontinuity result mentioned above any section of a line bundle $D_{\mid F}$ comes from a section in $D\left(f^{-1}(U)\right)$ for some open $U \subset \mathbf{P}^{2}$ containing $f(F)$. So Supp $\operatorname{Coker}\left(f^{*} f_{*} \mathcal{O}_{X}(D) \rightarrow \mathcal{O}_{X}(D)\right)$ does not contain any point in any of these fibres and therefore has codimension at least 2. Therefore the class $[D]$ belongs to the $f$-movable cone. To complete the proof we observe that any class in the cone $\left\{x \in N^{1}\left(X / \mathbf{P}^{2}\right): x \cdot F>0, x \cdot F_{i}^{a} \geq 0\right.$ for all $\left.a, i\right\}$ is the limit of classes $\left[D_{\alpha}\right]$ with $\left[D_{\alpha}\right] \cdot F_{i}^{a}>0$ for all $a$ and $i$. We have just proved that each class $\left[D_{\alpha}\right]$ belongs to the closed cone $\overline{M\left(X / \mathbf{P}^{2}\right)}$ and therefore so does their limit $x$. QED

We use this description of ${\overline{M\left(X / \mathbf{P}^{2}\right)}}^{e}$ together with Lemma 4.5 to find a rational polyhedral cone whose translates by the Mordell-Weil group cover the relative movable cone:

Lemma 4.7 There is a rational polyhedral subcone $K$ of $\overline{M\left(X / \mathbf{P}^{2}\right)}$ esuch that $\operatorname{Pic}^{0}\left(X_{\eta}\right) \cdot K=$ $\overline{M\left(X / \mathbf{P}^{2}\right)}$.

Proof: Let $W^{\prime}\left(X / \mathbf{P}^{2}\right)$ denote the affine subspace $\left\{y \in N^{1}\left(X / \mathbf{P}^{2}\right): y \cdot F=1\right\}$ and denote by $q$ the quotient map $W^{\prime}\left(X / \mathbf{P}^{2}\right) \rightarrow W\left(X / \mathbf{P}^{2}\right)$. By definition of the quotient action of $\operatorname{Pic}^{0}\left(X_{\eta}\right)$, for any $\phi \in \operatorname{Pic}^{0}\left(X_{\eta}\right)$ and $x \in N^{1}\left(X / \mathbf{P}^{2}\right)$ we have $\phi(q(x))=q(\phi(x))$. By Lemma 4.5 the action of $\mathrm{Pic}^{0}\left(X / \mathbf{P}^{2}\right)$ on $W\left(X / \mathbf{P}^{2}\right)$ has fundamental domain a rational polyhedron $\Pi$, and hence for the action on $W^{\prime}\left(X / \mathbf{P}^{2}\right)$ we have $\operatorname{Pic}^{0}\left(X / \mathbf{P}^{2}\right) \cdot q^{-1}(\Pi)=W\left(X / \mathbf{P}^{2}\right)$. Since the action of $\operatorname{Pic}^{0}\left(X / \mathbf{P}^{2}\right)$ preserves the $f$-effective $f$-movable cone, we can intersect with that cone on both sides to get $\operatorname{Pic}^{0}\left(X / \mathbf{P}^{2}\right) \cdot\left(q^{-1}(\Pi) \cap \overline{M\left(X / \mathbf{P}^{2}\right)}\right)^{e}=\overline{M\left(X / \mathbf{P}^{2}\right)}{ }^{e} \cap W\left(X / \mathbf{P}^{2}\right)$. Finally since $\operatorname{Pic}^{0}\left(X / \mathbf{P}^{2}\right)$ acts linearly we can multiply on both sides by positive scalars to get $\left.\operatorname{Pic}^{0}\left(X / \mathbf{P}^{2}\right) \cdot \mathbf{R}_{+}\left(q^{-1}(\Pi) \cap \overline{M\left(X / \mathbf{P}^{2}\right)}{ }^{e}\right)=\overline{M\left(X / \mathbf{P}^{2}\right.}\right)^{e}$. So taking $K=\mathbf{R}_{+}\left(q^{-1}(\Pi) \cap \overline{M\left(X / \mathbf{P}^{2}\right)}{ }^{e}\right)$ it remains to show that $q^{-1}(\Pi) \cap \overline{M\left(X / \mathbf{P}^{2}\right)}$ is a rational polyhedron in $W^{\prime}\left(X / \mathbf{P}^{2}\right)$. Since $\Pi$ is a rational polyhedron and by Lemma 4.6 the cone $\overline{M\left(X / \mathbf{P}^{2}\right)}{ }^{e}$ is defined by a finite set of inequalities, we need to show that $q^{-1}(\Pi) \cap{\overline{M\left(X / \mathbf{P}^{2}\right)}}^{e}$ is bounded. Choosing a section $s$ of $q$ we can write $W^{\prime}\left(X / \mathbf{P}^{2}\right)=V\left(X / \mathbf{P}^{2}\right)+\operatorname{im} s$. Let $\Pi^{\prime}$ denote the polyhedron $s(\Pi)$ : then $q^{-1}(\Pi)=V\left(X / \mathbf{P}^{2}\right)+\Pi^{\prime} \subset W^{\prime}\left(X / \mathbf{P}^{2}\right)$. So suppose a vector $v+s$ with $v \in V\left(X / \mathbf{P}^{2}\right)$ and $s \in \Pi^{\prime}$ belongs to $q^{-1}(\Pi) \cap{\overline{M\left(X / \mathbf{P}^{2}\right)^{e}}}^{e}$. By Lemma 4.6 the intersection numbers $(v+s) \cdot F_{i}^{a}$ must be nonnegative for all $i$ and $j$. Now $s \cdot F_{i}^{a}$ is bounded for $s \in \Pi^{\prime}$ by compactness of $\Pi^{\prime}$, so $v \cdot F_{i}^{a}$ is bounded below for all $a$ and $i$. But $v \cdot F_{j}^{1}=-v \cdot F_{j}^{2}$ for any $v \in V(X / S)$, therefore $v \cdot F_{i}^{a}$ is bounded above and below. If we write $v=\sum a_{l}^{k} D_{l}^{k}$ we have $v \cdot F_{i}^{a}=-2 a_{j}^{i}$ so the 
coefficients of $v$ are bounded. So the subset $q^{-1}(\Pi) \cap{\overline{M\left(X / \mathbf{P}^{2}\right)}}^{e}$ is bounded, hence rational polyhedral, as required. QED

So far we have considered the action of the Mordell-Weil group on the relative movable cone. To complete the proof, we lift our result to the absolute movable cone $\overline{M(X)}{ }^{e} \subset N^{1}(X)$. Precisely, we use the previous lemma to find a polyhedral cone in $\overline{M(X)}^{e}$ whose translates by the Mordell-Weil group cover the whole cone. Applying Theorem 3.7 we will see this implies the existence of a rational polyhedral fundamental domain, thereby completing the proof of the second part of Theorem 0.1 .

We note that so far everything we have said in this section applies to nets of arbitrary Mordell-Weil rank. Only now do we restrict to the case of Mordell-Weil rank $\rho=7$.

Lemma 4.8 Suppose $X$ is the blowup of $\mathbf{P}^{3}$ in the base locus of a net of quadrics which has 8 distinct basepoints, with Mordell-Weil rank $\rho=7$. Then $\overline{M\left(X / \mathbf{P}^{2}\right)}$ is the open half-space $\left\{[D] \in N^{1}\left(X / \mathbf{P}^{2}\right):[D] \cdot F>0\right\}$. Moreover, let $\Pi$ denote the rational polyhedron

$$
\Pi=\left\{\sum_{i} \alpha_{i}\left[E_{i}\right]: 0 \leq \alpha_{i} \leq 1 \text { for } i=2, \ldots, 8 ; \sum_{i} \alpha_{i}=1\right\} .
$$

contained in the affine hyperplane $W^{\prime}\left(X / \mathbf{P}^{2}\right)=\left\{[D] \in N^{1}\left(X / \mathbf{P}^{2}\right):[D] \cdot F=1\right\}$. Then $K=\mathbf{R}_{+} \Pi$ is a rational polyhedral cone satisfying the conclusion of Lemma 4.7; that is, $\operatorname{Pic}^{0}\left(X_{\eta}\right) \cdot K={\overline{M\left(X / \mathbf{P}^{2}\right)}}^{e}$.

Proof: The first claim follows directly from Lemma 4.6, since if the Mordell-Weil rank equals 7 there are no vertical divisors on $X$ other than multiples of $-\frac{1}{2} K_{X}$.

For the second claim Lemma 4.5 tells us that $\operatorname{Pic}^{0}\left(X_{\eta}\right)$ acts on the affine hyperplane $W^{\prime}\left(X / \mathbf{P}^{2}\right)$ as a group of translations. Now $\operatorname{Pic}^{0}\left(X_{\eta}\right)$ has a subgroup $G$ of finite index generated by the elements $E_{j}-E_{1}(j=2, \ldots, 8)$. It is clear that $\Pi$ is a fundamental domain for the action of $G$ on $W^{\prime}\left(X / \mathbf{P}^{2}\right)$. Furthermore, $W^{\prime}\left(X / \mathbf{P}^{2}\right)$ generates $\overline{M\left(X / \mathbf{P}^{2}\right)}$ as a cone, so by linearity of the action of the Mordell-Weil group, $K=\mathbf{R}_{+} \Pi$ is a fundamental domain for the action of $G$ on $\overline{M\left(X / \mathbf{P}^{2}\right)}$. In particular $G \cdot K={\overline{M\left(X / \mathbf{P}^{2}\right)}}^{e}$, which implies that $\operatorname{Pic}^{0}\left(X_{\eta}\right) \cdot K={\overline{M\left(X / \mathbf{P}^{2}\right)}}^{e}$ as required.

Theorem 4.9 Suppose $X$ is the blowup of $\mathbf{P}^{3}$ in the base locus of a net of quadrics which has 8 distinct basepoints, with Mordell-Weil rank $\rho=7$. There exists a rational polyhedral cone $U \subset \overline{M(X)}^{e}$ such that $\operatorname{Pic}^{0}\left(X_{\eta}\right) \cdot U=\overline{M(X)}^{e}$.

Proof: The first step is to choose a rational polyhedral cone $K_{0}$ in $\overline{M(X)}^{e}$ which maps onto the cone $K$ from the previous lemma. To do this, we observe that the extremal rays of $K$ are spanned by the vertices of the polyhedron $\Pi$, which are vectors of the form $\sum_{i \in I}\left[E_{i}\right]-(|I|-$ $1)\left[E_{1}\right]$, for $I$ any subset of $\{2, \ldots, 8\}$. Denote these vectors by $\left[v_{1}\right], \ldots,\left[v_{n}\right]$. For each vector $\left[v_{i}\right]$ we choose a preimage $v_{i}$ in $N^{1}(X)$ as follows. Suppose $\left[v_{i}\right]=\sum_{i \in I}\left[E_{i}\right]-(|I|-1)\left[E_{1}\right]$, and put $w_{i}=\sum_{i \in I} E_{i}-(|I|-1) E_{1}$. For each $\left[v_{i}\right]$, there is an element $\psi_{i} \in \operatorname{Pic}^{0}\left(X_{\eta}\right)$ such that $\psi_{i}\left(\left[v_{i}\right]\right)=\left[E_{1}\right]$. Therefore in $N^{1}(X)$, we have $\psi_{i}\left(w_{i}\right)=E_{1}+\frac{m_{i}}{2} K_{X}$ for some integer $m_{i}$. Since the class $-\frac{1}{2} K_{X}$ is preserved by $\operatorname{Pic}^{0}\left(X_{\eta}\right)$, this gives $\psi_{i}\left(w_{i}-\frac{m_{i}+1}{2} K_{X}\right)=E_{1}+\frac{1}{2} K_{X}$. We then define $v_{i}$ to be $w_{i}-\frac{m+1}{2} K_{X}$ for $m_{i}$ chosen as above. The point of this definition is that each vector $v_{i}$ then belongs to $\overline{M(X)}^{e}$. For this cone is preserved by pseudo-automorphisms, so it is enough to show that $\psi_{i}\left(v_{i}\right)=E_{1}+\frac{1}{2} K_{X}$ belongs to the cone. But this is straightforward: 
our calculation of $\overline{\operatorname{Curv}(X)}$ in the previous section shows that $E_{1}+\frac{1}{2} K_{X}$ is nef and hence semi-ample on $X$, so in particular it belongs to $\overline{M(X)}^{e}$. Finally we put $v_{0}=-\frac{1}{2} K_{X}$ (which also belongs to $\overline{M(X)}^{e}$ ) and define $K_{0}$ to be the rational polyhedral cone in $N^{1}(X)$ spanned by $\left\{v_{0}, \ldots, v_{n}\right\}$. We have shown that each extremal ray of $K_{0}$ lies in the cone $\overline{M(X)}$, so the whole cone $K_{0}$ does, and by construction $K_{0}$ maps onto $K$.

Now choose an SQM $X^{\prime}$ of $X$ and a divisor $D$ in the ample cone $A\left(X^{\prime}\right)$. By Lemma 4.7 there exists a divisor $D_{0}$ in $K_{0}$ and an element $\phi \in \operatorname{Pic}^{0}\left(X_{\eta}\right)$ such that $\phi_{*}\left(\left[D_{0}\right]\right)=[D]$ in $N^{1}\left(X / \mathbf{P}^{2}\right)$. Therefore in $N^{1}(X)$ we have $\phi_{*}\left(D_{0}\right)=D+\frac{m}{2} K_{X}$ for some $m$. If $m \leq 0$ then $D+\frac{m}{2} K_{X}$ is also ample on $X^{\prime}$. If $m>0$ then $\phi_{*}\left(D_{0}-\frac{m}{2} K_{X}\right)=D$ and since $D_{0}$ belongs to $K_{0}$ so too does $D_{0}-\frac{m}{2} K_{X}$. Therefore the union of all translates of $K_{0}$ by elements of $\operatorname{Pic}^{0}\left(X_{\eta}\right)$ intersects the interior of every nef cone inside $\overline{M(X)}^{e}$.

Finally $K_{0}$ is a rational polyhedral cone in $\overline{M(X)}^{e}$ so is contained in the union $U$ of finitely many nef cones $\overline{A\left(X^{\prime}\right)}$, each of which is rational polyhedral by Theorem 3.5. Since by Lemma 4.3 pseudo-automorphisms permute the nef cones of small modifications of $X$, the union of all the translates of $U$ by elements of $\operatorname{Pic}^{0}\left(X_{\eta}\right)$ is a union of nef cones. By the last paragraph this union intersects the interior of every nef cone, hence equals the whole effective movable cone $\overline{M(X)}^{e}$. QED

Corollary 4.10 Suppose $X$ is the blowup of $\mathbf{P}^{3}$ in the base locus of a net of quadrics which has 8 distinct basepoints, with Mordell-Weil rank $\rho=7$. Then there is a finite polyhedral fundamental domain for the action of PsAut* $(X, \Delta)$ or PsAut $(X)$ on $\overline{M(X)}{ }^{e}$.

Proof: If $U$ is the rational polyhedral cone of Theorem 4.9, we saw that $\overline{M(X)}^{e}=\operatorname{Pic}^{0}\left(X_{\eta}\right)$. $U$, hence $\overline{M(X)}^{e}=G \cdot U$ for $G$ equal to $\operatorname{PsAut}^{*}(X, \Delta)$ or $\operatorname{PsAut}^{*}(X)$. Taking $C$ to be the interior of $\overline{M(X)}$, this implies in particular that $C \subset G \cdot U$. Theorem 3.7 then says that $C_{+}=G \cdot U=\overline{M(X)}^{e}$, and there is a rational polyhedral fundamental domain for the action of $G$ on $C_{+}$. QED

This completes the proof of the second statement in Theorem 0.1 .

\section{References}

[1] C. Birkar, P. Cascini, C. Hacon, J. McKernan. Existence of minimal models for varieties of log general type. J. Amer. Math. Soc., to appear.

[2] R. Hartshorne. Algebraic Geometry. Springer (1977).

[3] Y. Kawamata. On the cone of divisors of Calabi-Yau fiber spaces. Internat. J. Math. 8 (1997), 665-687.

[4] J. Kollár, S. Mori. Birational Geometry of Algebraic Varieties. Cambridge University Press (1998).

[5] R. Lazarsfeld. Positivity in Algebraic Geometry. I. Springer (2004).

[6] E. Looijenga. Discrete automorphism groups of convex cones of finite type. arXiv:0908.0165.

[7] S. Mori. Threefolds whose canonical bundles are not numerically effective. Ann. of Math. 116 (1982), 133-176. 
[8] D. Morrison. Compactifications of moduli spaces inspired by mirror symmetry. Journées de géométrie algébrique d'Orsay (Orsay, 1992). Astérisque 218 (1993), 243-271.

[9] Y. Namikawa. Periods of Enriques surfaces. Math. Ann. 270 (1985), 201-222.

[10] K. Oguiso. On the finiteness of fiber-space structures on a Calabi-Yau 3-fold. J. Math. Sci. 106 (2001), 3320-3335.

[11] H. Sterk. Finiteness results for algebraic K3 surfaces. Math. Z. 189 (1985), 507-513.

[12] B. Szendröi. Some finiteness results for Calabi-Yau threefolds. J. London Math Soc. 60 (1999), 689-699.

[13] B. Totaro. Hilbert's fourteenth problem over finite fields, and a conjecture on the cone of curves. Compositio Mathematica 144 (2008), no. 5, 1176-1198.

[14] B. Totaro. The cone conjecture for Calabi-Yau pairs in dimension two. Duke Math. J., to appear.

[15] H. Uehara. Calabi-Yau threefolds with infinitely many divisorial contractions. J. Math. Kyoto Univ. 44 (2004), 99-118.

[16] P.M.H. Wilson. Minimal models of Calabi-Yau threefolds. Classification of algebraic varieties (L'Aquila, 1992), 403-410.

DPMmS, Wilberforce Road, Cambridge CB3 0WB, United Kingdom

Leibniz Universität Hannover, Institut für Algebraische Geometrie, Welfengarten 1, D-30167 Hannover, Germany

Email address: artie@math.uni-hannover.de 